\title{
THE FABRICATION OF CASSAVA SILK FIBROIN-BASED COMPOSITE FILM WITH GRAPHENE OXIDE AND CHITOSAN QUATERNARY AMMONIUM SALT AS A BIODEGRADABLE MEMBRANE MATERIAL
}

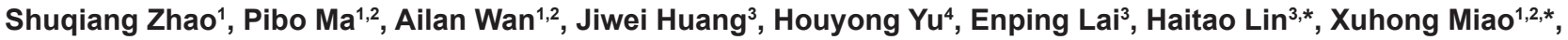 \\ Xinxia $\mathrm{Yue}^{3}$ \\ 1 Engineering Research Center for Knitting Technology, Ministry of Education, Jiangnan University, Wuxi 214122, China \\ 2 Key Laboratory of Eco-Textiles, Ministry of Education, Jiangnan University, Wuxi 214112, China \\ 3 Guangxi Key Laboratory of Sugar Resources of Green Processing \& School of Biological and Chemical Engineering, Guangxi University of Science and \\ Technology, Liuzhou 545006, China \\ 4The Key Laboratory of Advanced Textile Materials and Manufacturing Technology of Ministry of Education, College of Materials and Textiles, Zhejiang Sci- \\ Tech University, Hangzhou 310018, P. R. China \\ *Corresponding authors. E-mail: Ihthost@163.com; miaoxuhong@163.com
}

\begin{abstract}
:
A novel and excellent composite film was fabricated by simply casting cassava silk fibroin (CSF), chitosan quaternary ammonium salt (HACC), and graphene oxide (GO) in an aqueous solution. Scanning electron microscope images showed that when GO was dispersed in the composite films, the surface of CSF-based composite film became rough, and a wrinkled GO structure could be found. When the content of GO was $0.8 \%$, the film displayed a higher change with respect to the breaking strength and elongation, respectively, up to $97.69 \pm 3.69$ and $79.11 \pm 1.48 \mathrm{MPa}$, keeping good thermal properties because of the incorporation of GO and HACC. Furthermore, the novel CSF/ HACC/GO composite film demonstrates a lower degradation rate, implying the improvement of the resistance to the enzyme solution. Especially in the film with $0.8 \mathrm{wt} \% \mathrm{GO}$, the residual mass arrived at $64.35 \pm 1.1 \%$ of the primary mass after 21 days compared with the CSF/HACC film. This would reclaim the application of silk-based composite films in the biomaterial field.
\end{abstract}

\section{Keywords:}

CSF-based film, thermal properties, biodegradation membrane material, graphene oxide

\section{Introduction}

In recent years, some flexible composite films have allured sufficient attention due to their inherent properties comprising exceptional mechanical properties, remarkable incorruptibility [1], minimal flammability response, sufficient machinability, and suitable biodegradability [2] in the desirable biomaterial field. Silk fibroin (SF) is a naturally occurring fibrous protein synthesized from the posterior part of the silk gland. To date, SF obtained by degumming and dissolving has been devised and fabricated as films [3], scaffolds [4], hydrogels [5], microspheres, nanofibers, or non-woven mats [6] and has seen many distinctive applications. Especially SF-based composite films have been recognized as promising suitable biomaterials $[7,8]$, used in the skin [9], bone [10], blood vessel [11], nerve tissue, ligaments [12-14], and so on.

As we know, silk is divided into the domesticated bombyx mori silk [15], including mulberry silk and wild silkworm containing cassava silk [16], Antheraea pernyi silk [17], chestnut silk, and so on. Although bombyx mori silk fibroin (BSF) has been certified to be a popular replacement for conventional biomedical applications, the same cannot be said for cassava silk fibroin (CSF)-based composites, due to the limitations and lack of clarity with their morphology, thermal stability, and mechanical properties.

Earlier, the CSF membrane has been successfully fabricated by the layer-by-layer technique [18]. However, in practical applications, there are some defects about the mechanical property of SF membranes rendering them not suitable enough; also, the biodegradation rate compared with other biodegradable materials needs to be further ameliorated. Hence, the blending of CSF with other reinforcing polymeric materials to enhance the mechanical property of CSF-based composite materials [9] remains a challenge in the biomaterial field.

Recently, graphene oxide (GO) [19], one of the most excellent derivatives of graphene, has triggered remarkable attention owing to its specific structure, sufficient mechanical properties, and high exceptional surface area in several distinct applications $[20,21]$, including biomaterials. The two-dimensional carbon atom network of GO is composed of sp2 and sp3 connected to the oxygen-containing functional group's hybrid carbon atoms [22]. The surface of GO contains a large number of functional groups [23], such as hydroxyl and epoxy groups on the GO surface, and carboxyl groups at the edge of GO sheets. These functional groups actually endow GO with characteristic 
properties such as excellent hydrophilicity, suitable dispersion, and impressive biocompatibility. GO has considerable applications in electronic devices, reinforcing materials, biomaterials, and other fields, including drug delivery [22], cell affinity [24, 25], and enhancement of polymer nanomaterials due to its high specific surface area and abundant functional groups.

Moreover, some SF-based composite films [26] are prepared by the blending of the GO and BSF solution and have been studied previously [27]. Wang et al. [28] successfully prepared a flexible BSF/GO composite film using the solvent casting technique. When low concentrations of $\mathrm{GO}$ are incorporated into the SF solution, the characteristic properties of BSF/ GO composite film including mechanical properties, thermal stability, morphology, the resistance to enzymatic degradation are obviously improved. Meanwhile, the incorporation of GO contributes to the formation of a higher content of the silk II structure in the BSF/GO composite film. When low concentrations of GO are incorporated into the CSF/chitosan quaternary ammonium salt (HACC) solution, the properties (e.g., mechanical properties, thermal stability, and morphology) of the composite film are rarely reported, especially on the induction of the molecular conformation of CSF. This, therefore, has restricted their practical utilization as composite films in the biomaterial field.

In this work, a novel CSF/GO/HACC composite membrane with low content of GO is fabricated by a simple and green solvent casting method. It should be noted that the utilization of glutaraldehyde as a cross-linking agent of CSF and a certain concentration of GO in the CSF matrix are both essential to improve the molecular conformation of CSF. In contrast to previous researches, a comprehensive evaluation of the properties of the film including the morphology, thermal stability, and mechanical properties of CSF-based composite films has been carried out, thus extending the potential applications of SF-based composite films in the biomaterials used for skin.

\section{Materials and methods}

\subsection{Materials}

Raw cassava silk fibers were purchased from the Dandong border economic zone cooperation Baoli Industrial Co., Ltd. (Dandong, China). GO was purchased from Xfnano Materials Tech Co., Ltd. (Nanjing, China). Protease-XIV and glycerol were purchased from Sigma-Aldrich trading Co., Ltd. (Shanghai, China). HACC was purchased from Nantong Lushen Bioengineering Co., Ltd. (Nantong, China).

\subsection{The preparation of CSF solution}

A solution of SF was fabricated as previously described [29, 30] Briefly, raw cassava silk fibers were degummed thrice in boiling water with $0.05 \mathrm{wt} \% \mathrm{Na}_{2} \mathrm{CO}_{3}$ at a bath ratio of 1:50 for $30 \mathrm{~min}$, rinsed thoroughly, and dried at $60^{\circ} \mathrm{C}$ overnight. The extracted CSF fibers were dissolved in $9.3 \mathrm{~mol} \times \mathrm{L}^{-1} \mathrm{LiBr}$ solution by stirring at $60^{\circ} \mathrm{C}$ for $1.5 \mathrm{~h}$. The regenerated CSF solution was then dialyzed in a cellulose tube against deionized water for 4 days to cut off the salt debris. The resulting solutions were centrifuged at $9,000 \mathrm{rpm}$ for $20 \mathrm{~min}$ to remove the undissolved aggregates. The final concentrations of CSF solutions were adjusted to $3.5 \%(\mathrm{w} / \mathrm{v})$ with distilled water.

\subsection{Preparation of the CSF/HACC/GO composite films}

A suitable amount of HACC was dissolved in $2 \%(\mathrm{v} / \mathrm{v})$ acetic acid solution and magnetically stirred at room temperature overnight to prepare an HACC solution at $4 \mathrm{wt} \%$ with acetic acid. The diluted SF solution (2 wt\%), glycerol (0.3 wt\%), and glutaraldehyde $(0.02 \mathrm{wt} \%)$ were incorporated into the HACC acetic acid solution (4 wt\%) with acetic acid, respectively, dropwise. An amount of $100 \mathrm{mg} \mathrm{GO}$ powder was dispersed in $50 \mathrm{~mL}$ of distilled water by ultrasonication for $30 \mathrm{~min}$, resulting in the fabrication of a suitable $\mathrm{GO}$ solution $\left(2 \mathrm{mg} \times \mathrm{mL}^{-1}\right)$. Then sodium hydroxide solution $\left(1 \mathrm{~mol} \times \mathrm{L}^{-1}\right)$ was added to the $\mathrm{GO}$ solution to adjust its $\mathrm{pH}$ value to 10 . This $\mathrm{GO}$ solution was introduced into the CSF/HACC solution dropwise, to form a symmetrical and transparent blend solution by stirring magnetically for $40 \mathrm{~min}$. The final GO contents in the composite films were $0.2,0.4,0.6$, and $0.8 \mathrm{wt} \%$ against the weight of CSF, respectively. The composite films were poured into a glass Petri dish and kept in an oven at $40^{\circ} \mathrm{C}$ for $48 \mathrm{~h}$. Finally, they were rinsed with distilled water for $24 \mathrm{~h}$ to remove the residual glycerol and dried at room temperature.

\subsection{Thermal properties of CSF/HACC/GO composite films}

The thermal properties of the CSF/GO/HACC films were investigated by differential scanning calorimetry (DSC) and thermal gravimetric analysis (TGA). TGA curves were obtained on a TA Q600 instrument at a heating rate of $10^{\circ} \mathrm{C} \times \mathrm{min}^{-1}$ under a dry nitrogen gas flow of $50 \mathrm{~mL} \times \mathrm{min}^{-1}$. DSC was performed by a thermal analysis instrument (USA). The samples were heated from 0 to $600^{\circ} \mathrm{C}$ at $10^{\circ} \mathrm{C} \times \mathrm{min}^{-1}$ under a nitrogen atmosphere.

\subsection{Morphology and structure of CSF/HACC/GO composite films}

The surface morphologies of the samples were studied with a field-emission scanning electron microscope (SEM; JEOL, Japan). X-ray diffraction (XRD) curves of the CSF/GO films were studied using a Mini flex II (Rigaku, Japan) diffractometer with $\mathrm{Cu}$ Ka radiation.

All the samples were slit into microparticles before analysis and assessed with $2 q$ ranging from $5^{\circ}$ to $50^{\circ}$. The formation of Fourier transform infrared (FT-IR) spectra ( $\mathrm{KBr}$ pellet) on the films was studied on a Nicolet Magna-IR 750 spectrometer in the range of $4,000-500 \mathrm{~cm}^{-1}$.

\subsection{Behavior of CSF/HACC/GO composite films subjected to an enzyme reaction}

The blend films were incubated at $37^{\circ} \mathrm{C}$ in $50 \mathrm{~mL}$ of PBS (the common laboratory buffer) solution with $1 \mathrm{U} \times \mathrm{mL}^{-1}$ proteaseXIV for 5, 7, and 21 days. The enzyme solution was replenished with a fresh solution daily. The degradation rate was expressed 
as the percentage of weight loss relative to the initial dry weight. The residual samples were rinsed in distilled water and dried at $105^{\circ}$ at constant weight for further characterization [31]. The degradation rate was presented as the percentage of weight loss relative to the initial dry weight.

\section{Results and discussion}

\subsection{Morphology}

Figure 1 shows the surface and cross-sectional morphologies of three samples by SEM. Figure $1(\mathrm{~A})$ exhibits a typical thin crumpled silk veil wavy morphology, while an interesting relatively smooth and flat white nanoglobular morphology is observed for the CSF/HACC film, as shown in Figure 1(B). With the incorporation of GO nanosheets, the surface of the CSF/HACC/GO composite film became rough, and a wrinkled structure of GO could be observed, as shown in Figure 1(C), indicating the optimum dispersion without aggregation and the excellent interactions between the three components in the films [28].

\subsection{Structural characteristics}

The secondary structure of the CSF/HACC/GO composite films was determined by $X R D$ and FT-IR. Figure 2(b-f) illustrates the XRD curves of the membranes. The characteristic XRD diffraction peak of CSF appeared at $11.8^{\circ}$ and $22.0^{\circ}$ which could be evidence of the a-helical structure; $16.5^{\circ}, 20.2^{\circ}, 24$. $9^{\circ}, 30.90^{\circ}, 34.59^{\circ}$ were attributed to the b-sheet structure. As shown in Figure 2(a), the characteristic XRD peak of GO appeared at $2 q=10.6^{\circ}$, corresponding to a layer-to-layer distance (d-spacing) of $0.83 \mathrm{~nm}$ [32]. Both the CSF/HACC sample and the composite samples displayed prominent peaks at $20.01^{\circ}$ (Figure 2(b-f)), indicating a typical b-sheet structure. After GO was dispersed into the blend of CSF/HACC, the diffraction peak at $20.01^{\circ}$ [33] was gradually enhanced as the GO content increased to 0.2 and $0.8 \mathrm{wt} \%$ (Figure 2(c-f)). Moreover, the diffraction peak of $\mathrm{GO}$ at $10.50^{\circ}$ and $20.83^{\circ}$ [33] was discovered in the CSF/HACC/GO composite films.

Furthermore, Figure 3 illustrates the FT-IR spectra of the membranes. The infrared spectrum of CSF exhibited absorption band values of $1,640-1,615 \mathrm{~cm}^{-1}$ (amide I) attributable to the $\beta$-sheet structure, $3,291 \mathrm{~cm}^{-1}$ assigned to the random coil conformation, and $\mathrm{N}-\mathrm{H}$ stretching vibration of the protein molecule. Figure 3(a) illustrates the FT-IR spectra of $\mathrm{GO}$, exceptional absorption bands at 1,727.69, 1,619.78, and $1,046.02 \mathrm{~cm}^{-1}$, which were indicative of the $\mathrm{C}=\mathrm{O}$ stretching vibration of the carboxylic group, $\mathrm{C}=\mathrm{C}$ stretching vibration assigned to the $\mathrm{sp}^{2}$ network of the unoxidized graphite domains, and $\mathrm{C}-\mathrm{O}-\mathrm{C}$ stretching vibration of the epoxy group. Figure $3(b)$ shows the characteristic spectrum of the CSF/ HACC composite film and excellent absorption bands at $1,640.89 \mathrm{~cm}^{-1}$ was observed, demonstrating a typical $\beta$-sheet structure. Furthermore, the CSF/HACC composite film exhibited absorption bands at $1,063.02$ and $1,149.82 \mathrm{~cm}^{-1}$, which could be indicative of the characteristic vibration absorption peak of $-\mathrm{OH}$ in $\mathrm{HACC}$ and the stretching vibration of $\mathrm{C}-\mathrm{O}-\mathrm{C}$ bridge. Figure 3(c-f) shows the FT-IR spectra of the CSF/HACC/GO blend film. After GO was dispersed into the blend of CSF/ HACC, the peaks recorded $1,230.17 \mathrm{~cm}^{-1}$ (amide III $\beta$-sheet) a slight enhancement, implying that the molecular conformation of CSF transited from random coil/ $\alpha$-helix to $\beta$-sheet under the influence of GO. Meanwhile 1,063.02 $\mathrm{cm}^{-1}(-\mathrm{OH}$ absorption vibration) and $1,149.82 \mathrm{~cm}^{-1}$ (C-O-C stretching vibration) also slightly increased. Furthermore, the typical characteristic peaks at $1,727.69 \mathrm{~cm}^{-1}\left(\mathrm{C}=\mathrm{O}\right.$ stretching vibration), $1,619.78 \mathrm{~cm}^{-1}(\mathrm{C}=\mathrm{C}$ stretching vibration), and $1,046.02 \mathrm{~cm}^{-1}$ (C-O-C stretching vibration of the epoxy group) did not appear in the FT-IR

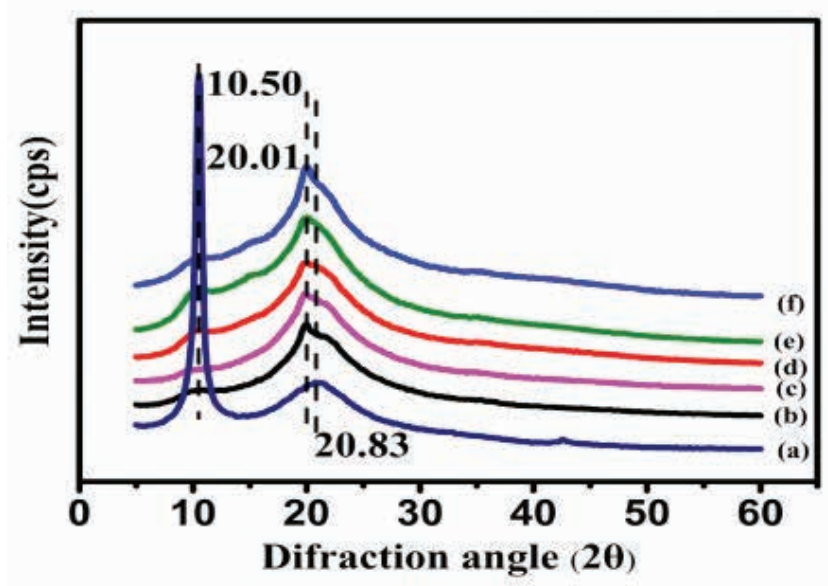

Figure 2. XRD curves of the CSF/HACC/GO films with different GO contents: (a) GO, (b) CSF/HACC, (c) CSF/HACC/GO $0.2 \mathrm{wt} \%$, (d) $\mathrm{CSF} / \mathrm{HACC} / \mathrm{GO} 0.4 \mathrm{wt} \%$, (e) CSF/HACC/GO $0.6 \mathrm{wt} \%$, and (f) CSF/ $\mathrm{HACC} / \mathrm{GO} 0.8 \mathrm{wt} \%$.

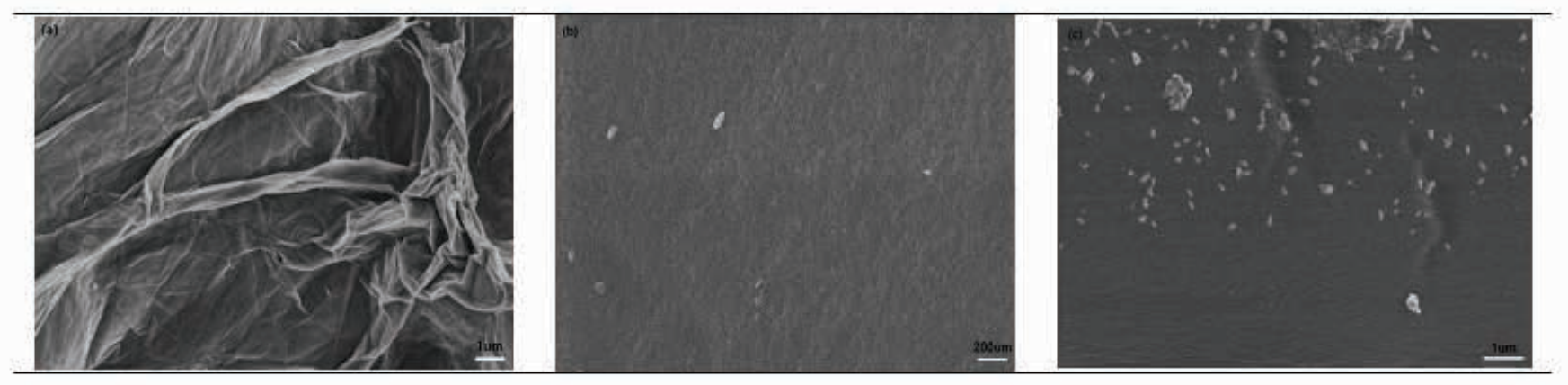

Figure 1. SEM images of the three samples: (A) surface of the GO, (B) surface of the CSF/HACC film, and (C) CSF/HACC/GO $0.8 \mathrm{wt} \% \mathrm{composite}$ film. 


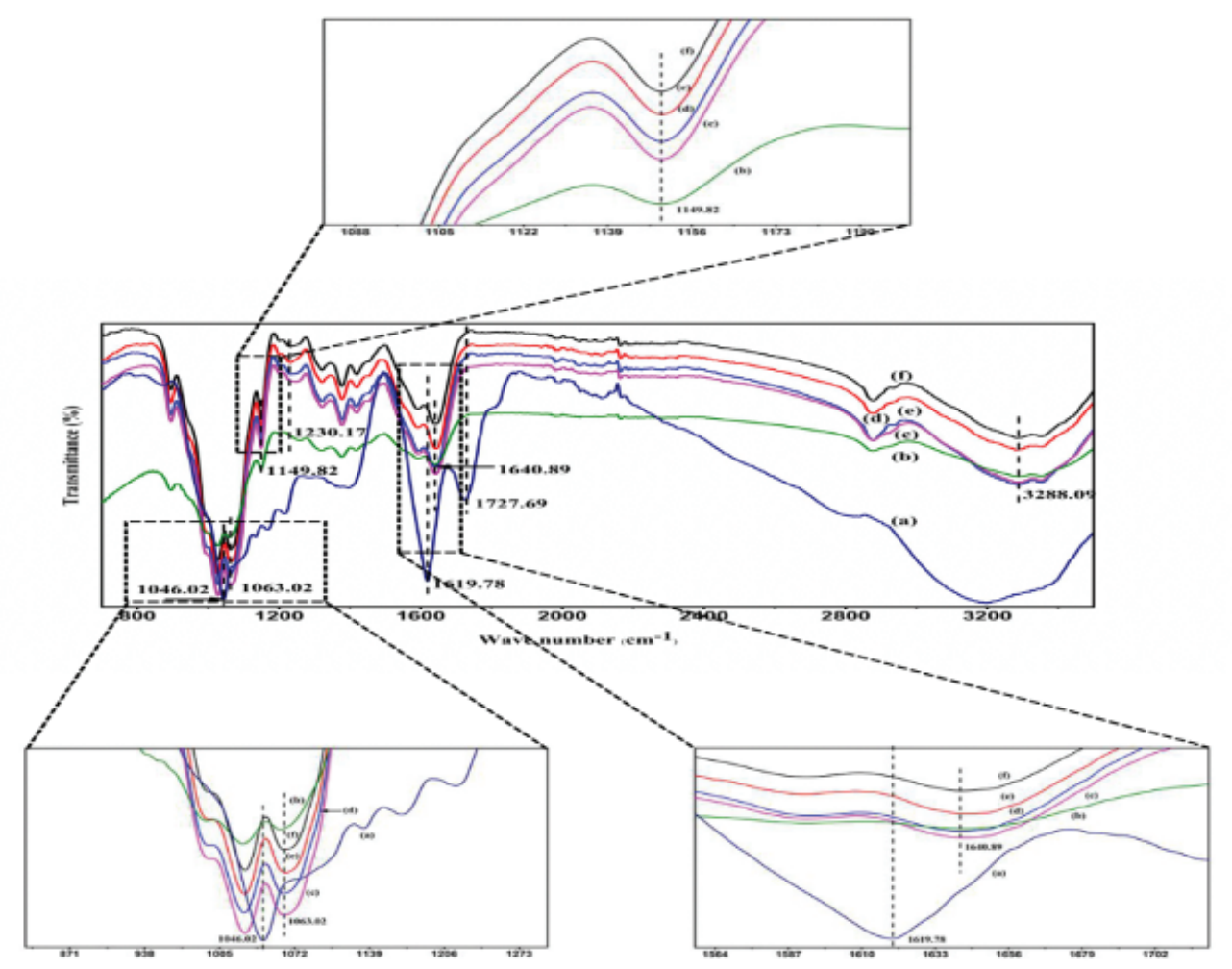

Figure 3. FT-IR curves of the CSF/HACC/GO films with different GO contents: (a) GO, (b) CSF/HACC, (c) CSF/HACC/GO 0.2 wt\%, (d) CSF/ $\mathrm{HACC} / \mathrm{GO} 0.4 \mathrm{wt} \%$, (e) CSF/HACC/GO $0.6 \mathrm{wt} \%$, and (f) CSF/HACC/GO0.8 wt $\%$.

spectra of CSF/HACC/GO composite films. It was likely that HACC modified the surface of GO, and the latter was evenly dispersed in the composite film.

\subsection{Thermal properties}

The thermal properties of the CSF/HACC/GO composite films were estimated by DSC and TGA. Figure 4 illustrates the TGA and DTG of the CSF/HACC sample and the composite samples. The TGA and DTG curves of the materials yielded three different features: the first was a weight loss which occurred at $60-100^{\circ} \mathrm{C}$ due to the evaporation of the bound water in the samples. Subsequently, the primary weight loss was from $190-300^{\circ} \mathrm{C}$ due to the thermal degradation of the volatile oxygen groups or the decomposition and melting of the amorphous zone of the internal structure of CSF with increase of temperature. The third weight loss was recorded above $300^{\circ} \mathrm{C}$ and this occurred due to the decomposition of more stable oxygen groups or the degradation of the crystallization portion with increasing temperature. When GO was dispersed in the composite films, both the CSF/HACC (Figure 4(b)) and the CSF/HACC/GO composite films (Figure 4(c-f)) exhibited homologous TGA and DTG curves. Figure 4(c-f) shows that the decomposition temperature improved with the increase of $\mathrm{GO}$ content. Especially when the content of $\mathrm{GO}$ was $0.8 \%$, the degradation temperature ran up to $287.86^{\circ} \mathrm{C}$, illustrating the higher thermal stability because of the strong intermolecular forces between the CSF or HACC molecular chains and the GO nanosheets compared with the CSF/HACC composite film.

Furthermore, Figure 5 shows the DSC curves of the CSF/HACC sample and the composite samples. All the samples yielded an endothermic peak at around $128^{\circ} \mathrm{C}$ due to the evaporation of the adsorbed water molecules in the samples. The primary degradation peaks were produced in the region of $290-320^{\circ} \mathrm{C}$ between the CSF/HACC film (Figure 5(b)) and CSF/HACC/ GO composite films (Figure 5(c-f)). With the incorporation of GO, Figure 5(c-f) shows that the decomposition temperature improved due to increase in the GO content compared with CSF/HACC composite film. When the content of GO was $0.8 \%$, the degradation temperature reached $305.27^{\circ}$, implying the increase in content of the $\beta$-sheet crystal structure [34] because of the incorporation of GO.

\subsection{Mechanical properties}

The mechanical properties of the SF-based composite film solutions were studied as previously described. GO was integrated into the CSF/HACC matrix to recompense for the mechanical properties [35], and to ensure that the fabricated CSF/HACC/GO-based biomaterial has sufficient mechanical properties as well as impressive toughness. The stressstrain curves of the SF films are shown in Figure 6, and the breaking strength and elongation at break are shown in Table 1. It could be observed that the breaking strength and elongation of the CSF/HACC film calculated were $40.24 \pm 2.96$ and $15.63 \pm 3.27 \mathrm{MPa}$, respectively. With the increase of the amount of GO into the composite films, the breaking strength and elongation gradually improved, the value arrived at $97.69 \pm 3.69$ and $79.11 \pm 1.48 \mathrm{MPa}$, respectively, at $0.8 \mathrm{wt} \%$ GO concentration. It was likely that some hydrogen bonds resulted between the CSF or HACC and GO. 

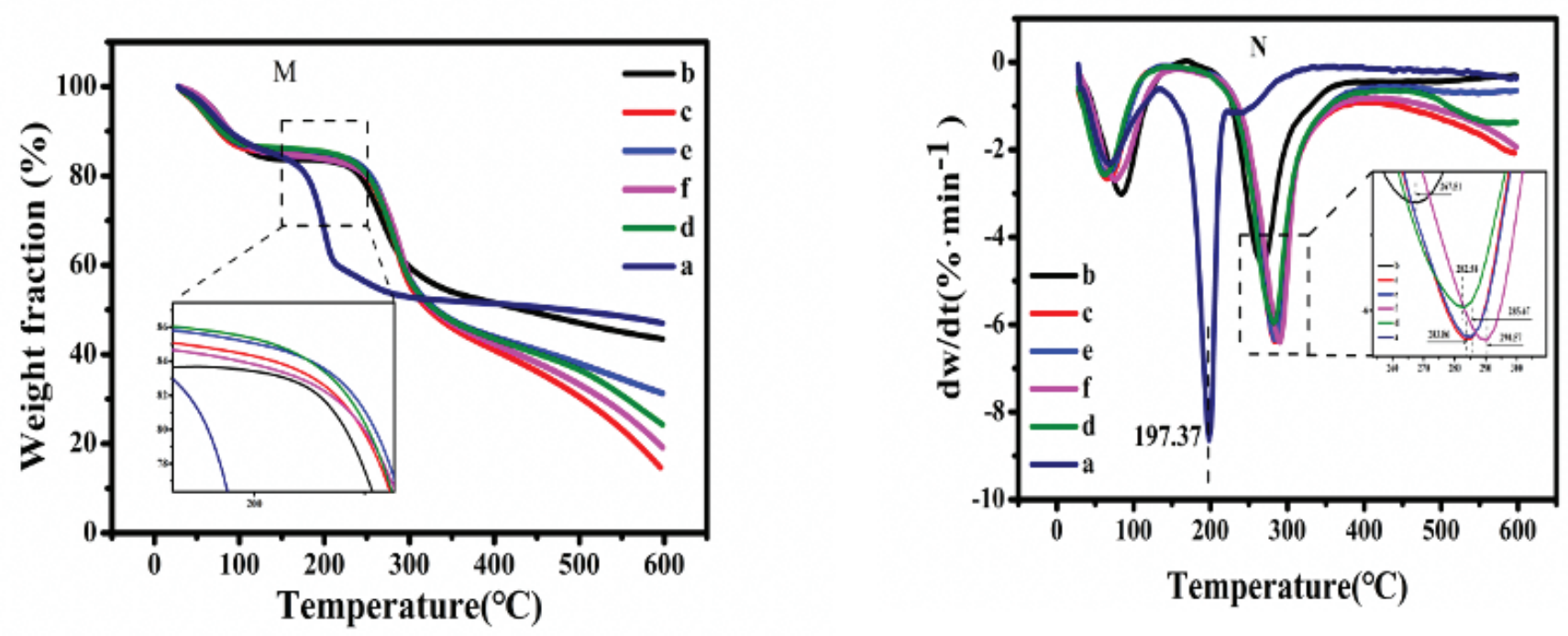

Figure 4. (M) TG and (N) DTG curves of the SF/HACC/GO films with different GO contents: (a) GO, (b) CSF/HACC, (c) CSF/HACC/GO 0.2 wt\%, (d) CSF/HACC/GO $0.4 \mathrm{wt} \%$, (e) CSF/HACC/GO $0.6 \mathrm{wt} \%$, and (f) CSF/HACC/GO0.8 wt\%.

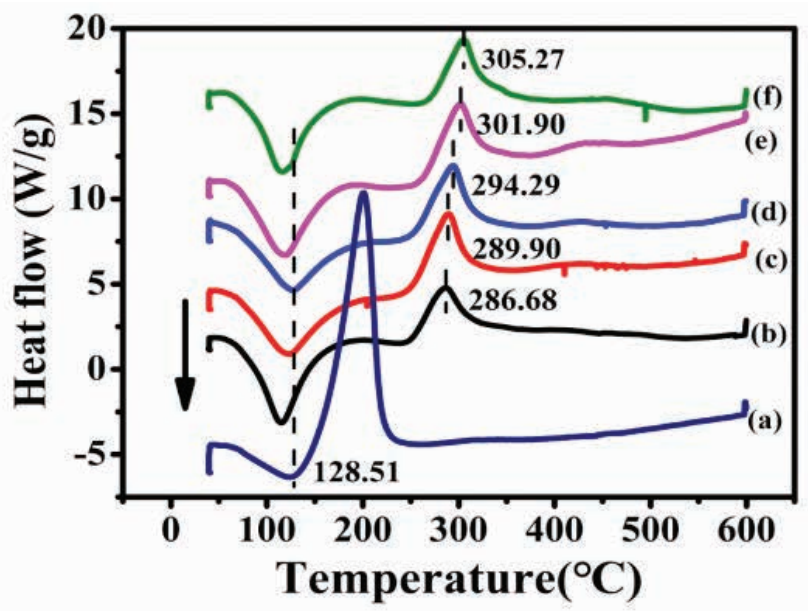

Figure 5. DSC curves of the CSF/HACC/GO films with different GO contents: (a) GO, (b) CSF/HACC, (c) CSF/HACC/GO $0.2 \mathrm{wt} \%$, (d) $\mathrm{CSF} / \mathrm{HACC} / \mathrm{GO} 0.4 \mathrm{wt} \%$, (e) CSF/HACC/GO $0.6 \mathrm{wt} \%$, and (f) CSF/ $\mathrm{HACC} / \mathrm{GO} 0.8 \mathrm{wt} \%$.

\subsection{Enzymatic degradation behavior of the SF/HACC/GO blend films}

The degradation behavior [36-38] was very significant in the biomaterial field. The degradation behavior of SF membrane was related to its secondary structure. In the degradation atmosphere of protease, the degradation rate decreased gradually with the increased content of the b-sheet structure.

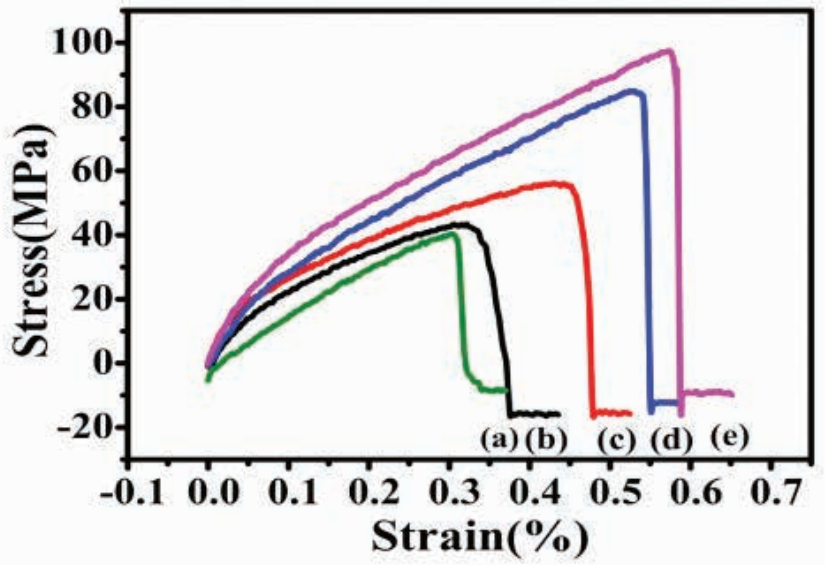

Figure 6. Stress-strain curves of the CSF/HACC/GO films with different GO contents: (a) CSF/HACC, (b) CSF/HACC/GO 0.2 wt $\%$, (c) CSF/HACC/GO $0.4 \mathrm{wt} \%$, (d) CSF/HACC/GO $0.6 \mathrm{wt} \%$, and (e) CSF/ HACC/GO0.8 wt\%.

In this study, CSF/HACC/GO blend films were fabricated to examine the effect of the blending ratio on the degradation behavior. Figure 7 shows the weight loss of the CSF/HACC/ GO blend films during a degradation trend over 21 days by protease-XIV. It was found that the CSF/HACC film rapidly degraded, and the residual mass was only $51.79 \pm 2.5 \%$ of the primary mass after 21 days. When GO was added to the composite films, the weight loss of the composite films

Table 1. The mechanical property of SF/HACC/GO composite films

\begin{tabular}{|c|c|c|}
\hline Sample & Breaking strength (MPa) & Elongation (\%) \\
\hline SF/HACC & $40.24 \pm 2.96$ & $45.63 \pm 3.27$ \\
\hline SF/HACC/GO (0.2 wt\%) & $43.74 \pm 4.54 \pm 5.73$ \\
\hline SF/HACC/GO (0.4 wt\%) & $55.46 \pm 7.32$ & $54.35 \pm 6.37$ \\
\hline SF/HACC/GO (0.6 wt\%) & $85.11 \pm 5.75$ & $68.92 \pm 2.45$ \\
\hline SF/HACC/GO (0.8wt\%) & $97.69 \pm 3.69$ & $79.11 \pm 1.48$ \\
\hline
\end{tabular}




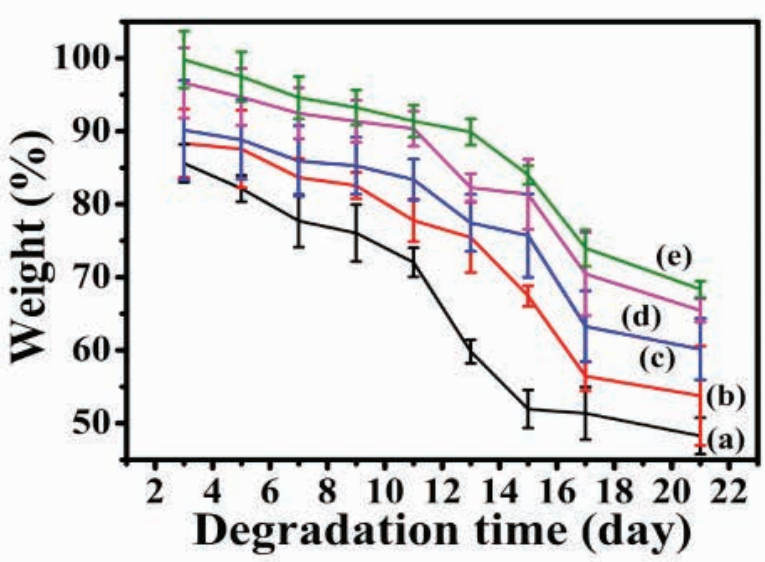

Figure 7. Enzymatic degradation behavior of the CSF/HACC/GO films with different GO contents: (a) CSF/HACC, (b) CSF/HACC/GO 0.2 wt $\%$, (c) CSF/HACC/GO $0.4 \mathrm{wt} \%$, (d) CSF/HACC/GO $0.6 \mathrm{wt} \%$, and (e) CSF/HACC/GO0.8 wt\%.

decreased gradually with time and the degradation rate was decreased with the increase of the amount of GO. Especially when the content of $\mathrm{GO}$ was $0.8 \%$, the residual mass was only $64.35 \pm 1.1 \%$ of the primary mass after 21 days (Figure $7(\mathrm{e})$ ). The incorporation of GO probably played an important role in enhancing the increase in the amount of the b-sheet crystal structure.

Furthermore, to exhibit the biodegradation behavior of the CSF/ HACC)/GO blend films, Figure 8 illustrates the SEM images of CSF/HACC/GO films after incubation for 5, 7, and 21 days in protease-XIV solution. It was observed that the surface of the CSF/HACC film was relatively smooth except for some white particles with uniform sizes (Figure 8(a)). With incubation in enzyme solution, prominent surface erosion was found on the surface of the CSF/HACC film after 5 days of degradation (Figure $8(A-1)$ ), and the degree of surface corrosion continued to be severe with increase in the incubation time (Figure $8(\mathrm{~A}-2$ and $A-3)$ ). Also, numerous debris were found within 21 days of incubation with enzyme solution (Figure 8(A-3)) compared with the degradation time of 7 days (Figure 8(A-2)). With the incorporation of $\mathrm{GO}$, the degree of surface erosion decreased with increase in the incubation time (Figure 8(B-1-B-3)), indicating the formation of more b-sheet crystal structures. It might also be that chemical crosslinking of regenerated CSF-based composite films played an important role in the biodegradability, including the degradation rate and degradation time of silk biomaterials. The CSF/HACC/GO composite membrane with $0.8 \mathrm{wt} \%$ GO concentration presented holeshaped residuals both within 5 days of degradation (Figure $8(\mathrm{~B}-1)$ ). And within 7 days (Figure $8(\mathrm{~B}-2)$ ) or 21 days (Figure $8(B-3)$ ) of incubation with enzyme solution (Figure $8(B-2)$ ), the films were still able to maintain the integrity of the membrane structure. All these implied that the molecular conformation of CSF changed from random coil/a-helix to b-sheet, suggestive of the GO component importantly improving with an impressive degradability of the blend films.

\section{Conclusions}

In sum, some flexible and exceptional CSF/HACC/GO composite films were fabricated by a simple and friendly technique without any harmful reagent. A higher content of $\beta$-sheet crystal structure in the blend of films was produced due to the noncovalent electrostatic interaction between groups with negative charges of GO and the groups with positive charges, and also owing to the intermolecular forces between the functional groups of GO and the polar groups on the SF molecular chains. The thermal stability and mechanical properties of the composite films gradually increased with the incorporation of certain concentrations of GO, which improved the biodegradability of silk biomaterials. This was the first time
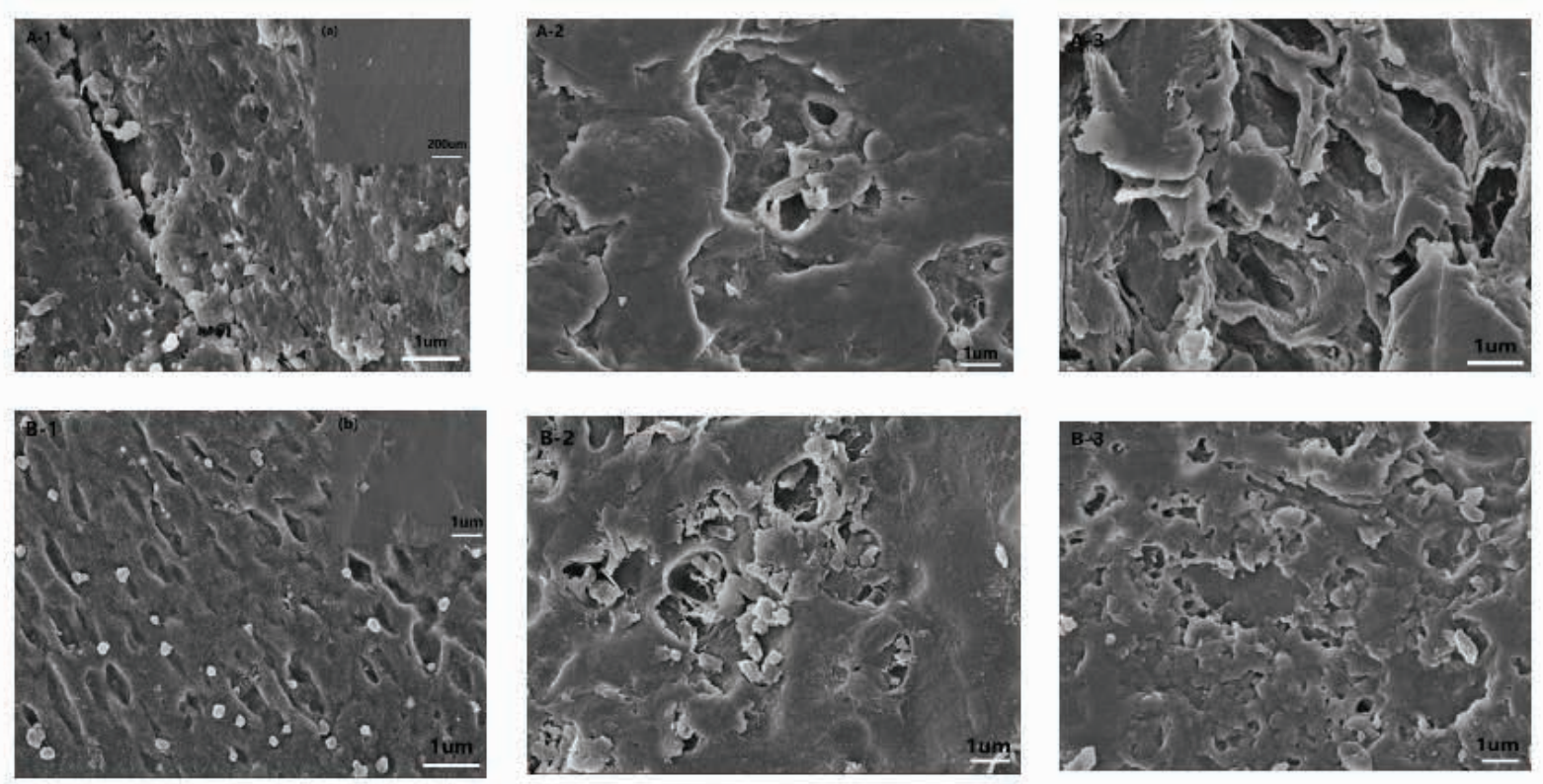

Figure 8. SEM images of CSF/HACC/GO films after incubation for 5,7 , and 21 days in protease-XIV solution. (A-1-A-3) the CSF/HACC films were degraded for 5, 7, and 21 days in protease-XIV solution, (B-1-B-3) the CSF/HACC/GO films were degraded for 5, 7, and 21 days in protease-XIV solution. (A) SEM images of the CSF/HACC films before degradation and (B) SEM images of the CSF/HACC/GO films before degradation. 
that CSF/HACC/GO-based composite films were developed. The resultant novel composite material was characterized by excellent biodegradability and has the potential for application in the field of biomaterials.

\section{ACKNOWLEDGMENTS}

The authors acknowledge financial support from the National Natural Science of China (11972172), the National Natural Science Foundation of China (51963002), and the Open Project Program of State Key Laboratory of Bio-Fibers and Eco-Textiles, Qingdao University (2017KFKT07).

\section{References}

[1] Oun, A. A., Rhim, J. W. (2015). Preparation and characterization of sodium carboxymethyl cellulose/cotton linter cellulose nanofibril composite films. Carbohydrate Polymers, 127, 101-109.

[2] Hu, X., Cebe, P., Weiss, A. S. Omenetto, F., Kaplan, D. L. (2012). Protein-based composite materials. Materials Today, 15(5), 208-215.

[3] Lu, Q., Hu, X., Wang, X. Q., Kluge, J. A., Lu, S. Z., et al. (2009). Water-insoluble silk films with silk I structure. Acta Biomaterialia, 6(4), 1380-1387.

[4] Nazarov, R., Jin, H. J., Kaplan, D. L. (2004). Porous 3-d scaffolds from regenerated silk fibroin. Biomacromolecules, 5(3), 718-726.

[5] Hopkins, A. M., Laporte, L. D., Tortelli, F., Spedden, E., Saii, C., et al. (2013). Silk hydrogels as soft substrates for neural tissue engineering. Advanced Functional Materials, 23(41), 5140-5149.

[6] Schneider, A., Wang, X. Y., Kaplan, D. L. (2009). Biofunctionalized electrospun silk mats as a topical bioactive dressing for accelerated wound healing. Acta Biomaterialia, 5(7), 2570-2578.

[7] Meinel, L., Fajardod, R., Hofmann, S., Langer, R., Chen, $J$. , et al. (2005). Silk implants for the healing of critical size bone defects. Bone (New York), 37(5), 688-698.

[8] Altman, G. H., Diaz, F., Jakuba, C., Calabro, T., Horan, $R$. L., et al. (2003). Silk-based biomaterials. Biomaterials, 24(3), 401-416.

[9] Zhang, Q., Zhao, Y. H., Yan, S. Q., Yang, Y. M., Zhao, H. J., et al. (2012). Preparation of uniaxial multichannel silk fibroin scaffolds for guiding primary neurons. Acta Biomaterialia, 8(7), 2628-2638.

[10] Miyamoto, S., Koyanagi, R., Nakazawa, Y., Nagano, A., Abiko, Y., et al. (2013). Bombyx mori silk fibroin scaffolds for bone regeneration studied by bone differentiation experiment. Journal of Bioscience \& Bioengineering, 115(5), 575-578.

[11] Lovett, M., Cannizzaro, C., Daheron, L., Messmer, B., Kaplan, D. L., et al. (2007). Silk fibroin microtubes for blood vessel engineering. Biomaterials, 28(35), 5271-5279.

[12] Thurber, A. E., Omenetto, F. G., Kaplan, D. L. (2015). In vivo bioresponses to silk proteins. Biomaterials, 71, 145157.

[13] Kundu, B., Rajkhowa, R., Kundu, S. C., Wang, X. (2007). Silk fibroin biomaterials for tissue regenerations. Advanced Drug Delivery Reviews, 65(4), 457-470.
[14] Li, X. F., Luo, R. C., Chen, Z. W., Li, G., Zhang, M. Z., et al. (2016). Silk fibroin scaffolds with a micro-/nano-fibrous architecture for dermal regeneration. Journal of Materials Chemistry B, 4(17), 2903-2912.

[15] Wang, L., Lu, C. X., Li, Y. H., Wu, F., Zhao, B., et al. (2015). Green fabrication of porous silk fibroin/graphene oxide hybrid scaffolds for bone tissue engineering. RSC Advance, 5(96), doi: 10.1039.78660-78668.

[16] Cebe, P., Partlow, B. P., Kaplan, D. L., Wurm, A., Zhuravlev, E., et al. (2015). Using flash DSC for determining the liquid state heat capacity of silk fibroin. Thermochimica Acta, 615, 8-14.

[17] Qian, S. W., Tang, Y., Li, X., Liu, Y., Zhang, Y. Y., et al. (2013). BMP4-mediated brown fat-like changes in white adipose tissue alter glucose and energy homeostasis. Proceedings of the National Academy of Sciences, 110(9), 798-807.

[18] Hu, K., Gupta, M. K., Kulkarni, D. D., Tsukruk, V. V. (2013). Ultra-robust graphene oxide-silk fibroin nanocomposite membranes. Advanced Materials, 25(16), 2301-2307.

[19] Ramanathan, T., Abdala, A. A., Stankocich, S., Dikin, D. A., Piner, R. D. et al. (2008). Functionalized graphene sheets for polymer nanocomposites. Nature Nanotechnology, 3(6), 327-331.

[20] Yun, H., Kim, M. K., Kwak, H. W., Lee, J. K., Kim, M. H., et al. (2013). Preparation and characterization of silk sericin/ glycerol/graphene oxide nanocomposite film. Fibers and Polymers, 14(12), 2111-2116.

[21]Xiao, L. Q., Liao, L. Q., Guo, X., Liu, L. J. (2013). One-pot synthesis of polyester-polyolefin copolymers by combining ring-opening polymerization and carbene polymerization. Macromolecular Chemistry \& Physics, 214(21), 25002506.

[22] Shan, C. S., Yang, H. F., Han, D. X., Zhang, Q. X., Lvaska, A., et al. (2010). Graphene/AuNPs/chitosan nanocomposites film for glucose biosensing. Biosensors \& Bioelectronics, 25(5), 1070-1074.

[23] Abdelsayed, V., Moussa, S., Hassan, H. M. A., Aluri, H., Collinson, M. M., et al. (2010). Photothermal deoxygenation of graphite oxide with laser excitation in solution and graphene-aided increase in water temperature. The Journal of Physical Chemistry Letters, 1(19), 2804-2809.

[24] Yang, H. P., Wang, L. Q., Zhao, J., Chen, Y. B., Lei, Z., et al. (2015). TGF- $\beta$-activated SMAD3/4 complex transcriptionally upregulates $\mathrm{N}$-cadherin expression in non-small cell lung cancer. Lung Cancer, 87(3), 249-257.

[25] Cui, R. L., Li, J., Huang, H., Zhang, M. Y., Guo, X. H., et al. (2015). Novel carbon nanohybrids as highly efficient magnetic resonance imaging contrast agents. Nano Research, 8(4), 1259-1268.

[26] Zhang, C., Zhang, Y., Shao, H., Hu, X. (2016). Hybrid silk fibers dry-spun from regenerated silk fibroin/graphene oxide aqueous solutions. ACS Applied Materials \& Interfaces, 8(5), 3349-3358.

[27] Wang, Y., Ma, R., Hu, K., Kim, S., Fang, G., et al. (2016). Dramatic enhancement of graphene oxide/ silk nanocomposite membranes: Increasing toughness and strength via annealing of interfacial structures. ACS Applied Materials \& Interfaces, 8(37), 24962-24973. 
[28] Wang, L., Lu, C. X., Zhang, B. P., Zhao, B., Wu, F., et al. (2014). Fabrication and characterization of flexible silk fibroin films reinforced with graphene oxide for biomedical applications. Royal Society of Chemistry Advances, 4(76), $40312-40320$.

[29] You, R. C., Xu, Y. M., Liu, Y., Li, X. F., Li, M. Z., et al. (2014). Comparison of the in vitro and in vivo degradations of silk fibroin scaffolds from mulberry and nonmulberry silkworms. Biomedical Materials, 10(1), 015003-015003.

[30] Huang, L., Li, C., Yuan, W. J., Shi, G. Q. (2013). Strong composite films with layered structures prepared by casting silk fibroin-graphene oxide hydrogels. Nanoscale, 5(9), 3780-3786.

[31] Li, X. F., Zhang, J., Feng, Y. F., Yan, S. Q., Zhang, Q., et al. (2018). Tuning the structure and performance of silk biomaterials by combining mulberry and non-mulberry silk fibroin. Polymer Degradation and Stability, 147, 57-63.

[32] Wang, S. D., Ma, Q., Wang, K., Ma, P. B. (2018). Strong and biocompatible three-dimensional porous silk fibroin/ graphene oxide scaffold prepared by phase separation. International Journal of Biological Macromolecules, 111(5), 237-246.
[33] Abdulkhani, A., Sousefi, M. D., Ashori, A., Ebrahimi, G. (2016). Preparation and characterization of sodium carboxymethyl cellulose/silk fibroin/graphene oxide nanocomposite films. Polymer Testing, 52(7), 218-224.

[34] Panda, N., Biswas, A., Sukla, L. B., Pramanik, K. (2014). Degradation mechanism and control of blended eri and tasar silk nanofiber. Applied Biochemistry \& Biotechnology, 174(7), 2403-2412.

[35] Lu, S. Z., Wang, X. Q., Lu, Q., Zhang, X. H., Kluge, J. A., et al. (2010). Insoluble and flexible silk films containing glycerol. Biomacromolecules, 11(1), 143-150.

[36] You, R. C., Xu, Y. M., Liu, G. Y., Liu, Y., Li, X. F., et al. (2014). Regulating the degradation rate of silk fibroin films through changing the genipin crosslinking degree. Polymer Degradation and Stability, 109(3), 226-232.

[37] Zhang, W., Ahluwalia, I. P., Literman, R., Literman, D. L., Yelick, P. C., et al. (2011). Human dental pulp progenitor cell behavior on aqueous and hexafluoroisopropanol based silk scaffolds. Journal of Biomedical Materials Research Part A, 97(4), 414-422.

[38] Loh, Q. L., Choong, B. C., Meng, D. M., Mimmm, C. (2013). Three-dimensional scaffolds for tissue engineering applications: Role of porosity and pore size. Tissue Engineering. Part B, Reviews, 19(6), 485-502. 